\title{
With or against Each Other? The Influence of a Virtual Agent's (Non)cooperative Behavior on User's Cooperation Behavior in the Prisoners' Dilemma
}

\author{
Carolin Straßmann $\mathbb{D D}^{1}{ }^{1}$ Astrid M. Rosenthal-von der Pütten, ${ }^{2}$ and Nicole C. Krämer ${ }^{1}$ \\ ${ }^{1}$ Social Psychology: Media and Communication, University Duisburg-Essen, 47057 Duisburg, Germany \\ ${ }^{2}$ Individual and Technology, RWTH Aachen University, 52062 Aachen, Germany \\ Correspondence should be addressed to Carolin Straßmann; carolin.strassmann@uni-due.de
}

Received 23 April 2018; Accepted 12 August 2018; Published 20 September 2018

Academic Editor: Thomas Mandl

Copyright (C) 2018 Carolin Straßmann et al. This is an open access article distributed under the Creative Commons Attribution License, which permits unrestricted use, distribution, and reproduction in any medium, provided the original work is properly cited.

\begin{abstract}
Most applications for virtual agents require the user to cooperate. Thus, it is helpful to investigate different strategies for virtual agents to evoke the user's cooperation. In the present work $(N=80)$, we experimentally tested the influence of an agent's (non)cooperative nonverbal behavior and actual decision-making behavior on user's cooperation in the Prisoners' Dilemma considering different age groups (students and seniors). Therefore, we used a 2 (nonverbal behavior) x 2 (age group) betweensubjects design in Wizard-of-Oz study. Results show age differences with seniors cooperating more often than students do. The nonverbal behavior had no effect on the users' willingness to cooperate nor on the evaluation of the agent's cooperativeness. However, the agent's decision-making behavior in the game influenced the users' willingness to cooperate. In summary, the nonverbal behavior seemed to be too subtle, while the actions of the agent were important in terms of cooperation.
\end{abstract}

\section{Introduction}

The long-term goal of most endeavors regarding virtual agents is to create engaging experiences and interactions that are beneficial for the user. In most scenarios, however, the success of these systems depends on the user's willingness to cooperate with the virtual agent. In some scenarios this is even more crucial, for instance, with regard to interactions that are essential for the user's health. Systems supporting elderly users in their daily life, organizing appointments, and reminding them to take their medication rely on users who are willing to share information in order to keep record of all appointments or to guarantee successful medicinal treatment (see [1]). The challenge is to create interactions in which users do not feel patronized by the system but to facilitate cooperation. Interlocutors can show cooperation via different channels: (a) via nonverbal behavior signaling the intent for cooperation, (b) via direct verbal messages, and (c) via their actual cooperative or noncooperative decision-making behavior.
Since there is ample evidence that specific nonverbal behavior can lead to higher cooperativeness perception [26], the present study focuses on the effects of the agent's nonverbal behavior. In most situations, it is risky to trust and cooperate with a dishonest person. In order to avoid putting trust into the wrong person, interlocutors use nonverbal behavior as indicators for commitment and cooperation [7]. Nonverbal behavior might function as an indicator for cooperation in such a subtle way that the agent can evoke users' willingness to cooperate without making them feel forced to cooperate. Boone and Buck [3] claimed that nonverbal expressiveness plays an important role in the cooperation process. The authors further suggest testing this assumption by integrating nonverbal emotional displays (nonverbal expressiveness) within a social dilemma setting (e.g., Prisoner's Dilemma). In line with this claim, prior research demonstrated that interlocutors are sensitive to subtle facial expressions like smiling when they evaluate the other person's cooperativeness [5] and that smiling can evoke more cooperation behavior [6]. It has been shown that people who 
are more cooperative showed more expressive facial displays (both positive and negative) [8]. These facial expressions are assumed to signal honest intentions to cooperate [2]. Thus, a smiling agent should be perceived to signal cooperativeness and therefore users are more willing to cooperate with this agent in return. The effect of expressiveness on the perception of cooperativeness is not limited to facial expressions, but also expressiveness in body movements (large and high frequent gestures) was found to elicit higher perceptions of cooperativeness [4]. Moreover, gaze behavior and headtilts are related to trust, a concept highly correlated to commitment and cooperation. Hence, gazing towards the user displaying a lateral head-tilt has also been found to evoke a more cooperative impression of the interlocutor [4].

In sum, prior findings suggest that specific nonverbal behavior (e.g., high expressiveness, gaze behavior, and headtilt) will lead to the attribution of cooperativeness, which recursively fosters the cooperation behavior of the interlocutor. Therefore, we hypothesize the following:

H1: Users interacting with an agent showing cooperative nonverbal behavior cooperate more often with the agent.

Besides the nonverbal behavior of interlocutors, their actual decision-making behavior in the Prisoner's Dilemma is of course also a crucial indicator for trust and cooperation. Pletzer and colleagues [9] found that people are more willing to cooperate when they expect their counterpart to cooperate. Hence, once the trust between the interlocutors has been destroyed by not being cooperative, the perceived cooperativeness of the counterpart will also decrease. Following this we assume that if the agent decides not to cooperate, users' cooperative behavior will drop in return.

H2: The agent's actual decision-making behavior in the Prisoner's Dilemma influences users' willingness to cooperate. After choosing not to cooperate, user's cooperative decision-making behavior will decrease.

As mentioned above, especial applications for special target groups like seniors (e.g., daily life assistance) require the user to cooperate. However, these target groups have special needs due to their features and possibilities. It has been shown, that elderly people are more nervous during an interaction with a virtual agent [10] and, thus, it is especially important to calm them down and find a subtle way to gain their willingness to cooperate. One potential factor to evoke cooperation behavior might be nonverbal cues, but prior findings suggest that seniors showed a higher tendency to misinterpret emotional displays [11]. Hence, it is possible that seniors might also show a higher tendency to misinterpret nonverbal cooperation indicators. Since nonverbal behavior is assumed to be a good natural way to induce cooperation [3], it is a matter of concern whether this is also the case for elderly people, who would particularly benefit from it. Thus, this research aims to investigate agerelated differences and the following research question will be examined:

RQ1: Are there age-related differences in the perception of the agent's cooperativeness and the users' willingness to cooperate?
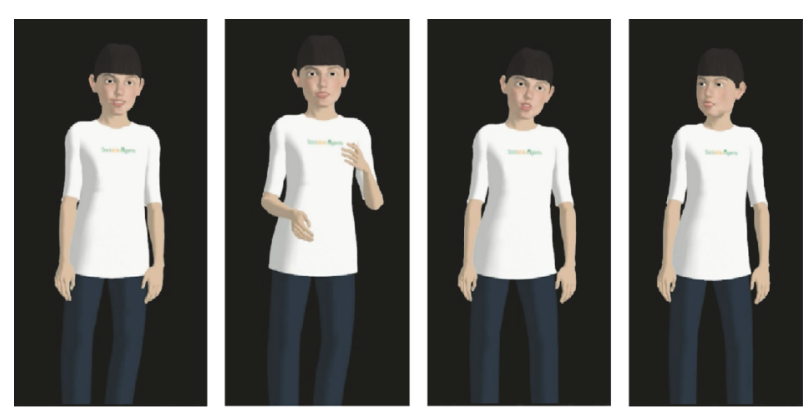

FIgURE 1: Examples of the presented nonverbal behavior smiling, expressive gesture, lateral head-tilt (cooperative nonverbal behavior), and avoiding gaze (noncooperative nonverbal behavior).

\section{Materials and Methods}

2.1. Experimental Design and Experimental Tasks. Depending on the experimental group, the virtual agent in this study showed either cooperative or noncooperative nonverbal behavior prior to and during the Prisoners' Dilemma game. The study was set up as Wizard-of-Oz scenario in a $2 \times 2$ between-subjects design with the independent factors "age group" (students versus seniors) and "nonverbal behavior" (cooperative versus noncooperative).

For the implementation of the two types of nonverbal behaviors, we rely on prior research [4] in which nonverbal behaviors were systematically tested with regard to how users perceived the virtual agent's expressivity and gaze behavior with regard to cooperativity. For the present study, we adopted these behaviors. Therefore, in the cooperative condition the agent ("Billie") showed expressive facial and body movements, turned the gaze towards the user, and showed a lateral head-tilt. Since expressiveness is one of the main indicators for cooperation, the agent in the noncooperative condition in contrast barely displayed nonverbal behavior and avoided the user's gaze more often. Figure 1 presents examples of these behaviors.

To test the impact of (non)cooperative nonverbal behavior we created situations in which cooperation is the key for the interaction. Participants played a virtual version of the well-established Prisoners' Dilemma (c.f. Figure 2). This version of the game is money-based. The players played four rounds in which both parties decided whether to place or to hold their money $(10 €)$ without knowing in advance the decision of the other player. The gains in this game depended on the mutual decision to cooperate: when both parties cooperated and placed their money, they both doubled their placement (both gain $20 €$ ). If just one player placed his or her money and the other player decided to hold, the cooperating player lost the placement $(0 €)$ and the other tripled his or her stakes $(30 €)$. When both players decided to hold their stakes nothing happens and both stakes remained (10 €). Within the four rounds, the virtual agent showed the same decisionmaking behavior for each participant: first, he cooperates, then he does not cooperate, and in the last two turns, he cooperates again. 


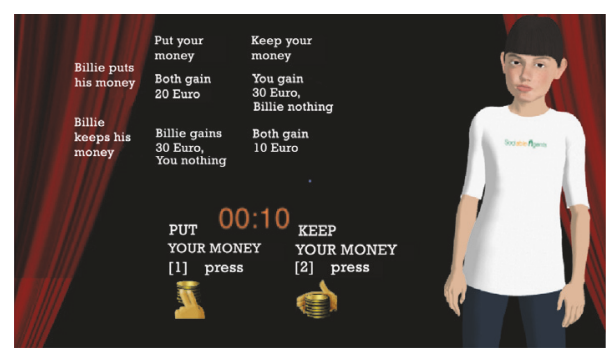

Figure 2: Prisoner's Dilemma.

Before participants played the Prisoner's Dilemma, they had the chance to get to know the agent. Participants interacted with the virtual agent in a calendar setting, where they managed appointments and decided whether to follow suggestions of the virtual agent. In particular, participants planned three appointments together with the virtual agent Billie and entered the appointments into the virtual calendar by stating activity as well as date, starting time, and duration of the appointment. During this interaction, the agent already displayed the nonverbal behavior (either cooperative or noncooperative), so that participants also had the chance to perceive the manipulated cooperativity of the agent during this joint task and prior to the dilemma task.

2.2. Participants and Procedure. Overall 80 people participated in this lab experiment, 40 of which were students (age: $M=22.03, S D=2.61 ; 24$ female and 16 male) and 40 seniors (age: $M=68.15, S D=5.59 ; 22$ female and 18 male). Students received either course credits or $10 €$ and seniors received $10 €$ for participation. Upon arrival, participants read and signed informed consent. Afterwards, they first interacted with the agent in the calendar setting negotiating appointments and filled in a short evaluating questionnaire, in which they evaluated the agent's person perception. Subsequently, they were instructed about the mechanisms of the Prisoners' Dilemma and then they played four rounds of the game. After completion of the game, participants filled in questionnaires evaluating the agent and its abilities, the interaction, and the experimental tasks as well as scales assessing the explanatory variables.

\subsection{Measurements}

2.3.1. Cooperation Behavior. We analyzed users' behavior within the Prisoners' Dilemma. Participants could choose to cooperate or not during each of the four turns. We also calculated the sum score for all four decisions.

2.3.2. Person Perception. Participants were asked to evaluate the agent with regard to person perception on five dimensions (Likability, Intelligence, Cooperativity, Dominance, and Autonomy), indicating their agreement to 35 items on a 5point Likert scale ranging from "I do not agree at all" to "I fully agree". The scales are based on a person perception scale previously used in human-agent/robot interaction studies [12]. Participants stated their evaluation of the agent's person perception twice: after the calendar interaction (T1) and after the Prisoners' Dilemma (T2). The dimension Autonomy was measured only once at $\mathrm{T} 2$.

The dimension Likability was measured with eight items (e.g., friendly, likable; Cronbach's $\alpha T 1=.911 ; T 2=.882$ ). Intelligence was measured using five items (e.g., dumb (rev), intelligent; Cronbach's $\alpha T 1=.820 ; T 2=.845)$. Cooperativity was measured using seven items (e.g., ask Billie for advice, is cooperative, is supportive; Cronbach's $\alpha T 1=.891$; $T 2$ $=.859$ ). Autonomy was measured using six items (e.g., not autonomous (rev), self-dependent; Cronbach's $\alpha$ T2 =.773). Dominance was measured using nine items (e.g., dominant, submissive (rev)). Reliability was too low for this subscale and did not improve when using a subset of the items. Therefore, we decided to use only the single item dominant for further analyses.

2.3.3. Communicative Abilities. We assessed how participants rated the agent's verbal and nonverbal communicative abilities with regard to mutual verbal understanding, dominance in conversation, nonverbal behavior production, and nonverbal behavior recognition/understanding. Mutual verbal understanding was measured with nine items (e.g., Billie understood me well, did not hear me, did not understand me; Cronbach's $\alpha=.757$; the item "the conversation with Billie was stiff" was omitted to enhance reliability). Dominance in conversation was measured using five items (e.g., I was able to control the conversation (rev), Billie was leading the conversation; Cronbach's $\alpha=.744$ ). Nonverbal behavior production was measured with five items (e.g., I had the feeling that Billie's gestures were expressive, unambiguous; Cronbach's $\alpha=.832$ ). Nonverbal behavior understanding was measured with six items (e.g., I had the feeling that Billie was able to recognize my gestures, was able to recognize my mimic; Cronbach's $\alpha=.888$ ).

2.3.4. Physical Presence and Social Presence. We assessed participants' sense of copresence with the Nowak and Biocca Presence Scale [13], which contains 12 items on the concept of "perceived other's copresence" (e.g., my interaction partner was intensely involved in our interaction; Cronbach's $\alpha=.784$ ) and 6 items on "self-reported copresence" (e.g., I was unwilling to share personal information with my interaction partner; Cronbach's $\alpha=.685$ ), both rated on a 5-point Likert scale. Furthermore, we used Bailenson et al.s Social Presence Scale [14] with 5 items rated on a 5-point Likert scale (e.g., I perceived the presence of another person in the room with me, Cronbach's $\alpha=.699$ ). Since reliability of the Social Presence Scale and the subscale "self-reported copresence" was low, we excluded these measures from further analyses.

2.3.5. Perception of the Game. We asked participants about their perceptions of Billie and the Prisoners' Dilemma with fifteen ad hoc generated items. These items asked participants, for example, about the difficulty of the game (e.g., the game was easy to master), the perceived cooperation of the game (e.g., Billie and I were partners during the game), the trust towards the agent (e.g., Billie gave me the feeling that he knows better and I am better off not to trust him), or felt competitiveness (e.g., during the game I was mostly 
TABLE 1: Means of participants' decision to cooperate for all four rounds.

\begin{tabular}{|c|c|c|c|c|c|c|c|c|c|}
\hline & \multicolumn{3}{|c|}{ Students } & \multicolumn{3}{|c|}{ Seniors } & \multicolumn{3}{|c|}{$\sum$} \\
\hline & $\mathrm{CO}$ & $\mathrm{NC}$ & $\sum$ & $\mathrm{CO}$ & $\mathrm{NC}$ & $\sum$ & $\mathrm{CO}$ & $\mathrm{NC}$ & $\sum$ \\
\hline \multicolumn{10}{|c|}{ Round $1_{\mathrm{a}, \mathrm{b}}$} \\
\hline$M$ & 0.90 & 0.75 & 0.83 & 0.90 & 1.00 & 0.95 & 0.90 & 0.88 & 0.89 \\
\hline$S D$ & 0.31 & 0.44 & 0.38 & 0.31 & 0.00 & 0.22 & 0.30 & 0.33 & 0.32 \\
\hline \multicolumn{10}{|c|}{ Round $2_{c, d}$} \\
\hline$M$ & 0.85 & 0.75 & 0.80 & 0.85 & 0.95 & 0.95 & 0.85 & 0.85 & 0.85 \\
\hline$S D$ & 0.37 & 0.44 & 0.41 & 0.37 & 0.22 & 0.30 & 0.37 & 0.37 & 0.36 \\
\hline \multicolumn{10}{|c|}{ Round $3_{\mathrm{a}, \mathrm{c}}$} \\
\hline$M$ & 0.70 & 0.50 & 0.60 & 0.80 & 0.65 & 0.73 & 0.75 & 0.58 & 0.66 \\
\hline$S D$ & 0.47 & 0.51 & 0.50 & 0.41 & 0.45 & 0.45 & 0.44 & 0.50 & 0.48 \\
\hline \multicolumn{10}{|c|}{ Round $4_{\mathrm{b}, \mathrm{d}}$} \\
\hline$M$ & 0.40 & 0.55 & 0.48 & 0.65 & 0.75 & 0.70 & 0.53 & 0.65 & 0.59 \\
\hline$S D$ & 0.50 & 0.51 & 0.51 & 0.49 & 0.44 & 0.46 & 0.51 & 0.48 & 0.50 \\
\hline
\end{tabular}

Note. Means in rows sharing subscripts are significantly different from each other. CO represents a cooperative nonverbal behavior, while NC is written for the noncooperative nonverbal behavior.

concerned with how I could maximize my own gains). All items were rated on a 5-point Likert scale ranging from "I do not agree at all" to "I do fully agree".

2.3.6. Future Usage of Agent System. Participants also indicated how likely they would use the virtual agent in the future (e.g., I can imagine interacting with Billie more often, Billie could help me also with other tasks in my everyday life; Cronbach's $\alpha=.955$ ).

2.3.7. Moderating Variables. As moderating variables, we used the short version of the NEO-FFI Scale [15], the cooperation subscale of the Temperament and Personality Questionnaire [16], and the nonverbal immediacy scale [17].

\section{Results}

3.1. Decision-Making Behavior. A mixed-design repeated measures ANOVA was calculated with the agent's nonverbal behavior and age groups as between factors and users decision-making behavior during the game (user's decision to cooperate or not within the four rounds) as repeated measures. Greenhouse-Geisser correction $(\varepsilon=.78)$ was used, since the assumption of sphericity has been violated. No main effect of the presented nonverbal behavior emerged, while there was a significant main effect for the age group $(F(1,76)=$ $\left.9.438, p=.003, \eta^{2}=.110\right)$ and a linear effect of the cooperation behavior itself $\left(F(2.35,178.63)=9.852, p<.001, \eta^{2}=.115\right)$. Post hoc tests indicated that participants cooperated in the first and second round significantly more often compared to the third and fourth round (cf. Table 1). Considering the overall cooperation behavior, an ANOVA with age and nonverbal behavior as independent and the frequency of cooperation (ranging from 0 to 4 ) as dependent variable was conducted. Analyses revealed only a significant difference regarding age $\left(F(1,76)=9.438, p=.003, \eta^{2}=.110\right)$ : seniors cooperated more often than students (cf. Table 2).
3.2. Person Perception of Agent. Multiple ANOVAs were calculated, to investigate the effect of the nonverbal behavior and age group on the five subscales of person perception (after the interaction) revealing no significant effects on Cooperativity, Competence, and Autonomy. However, a significant main effect of the agent's nonverbal behavior on Likability was found, $F(1,79)=5.04, p=.028, \eta^{2}=.062$. An agent showing noncooperative nonverbal behavior was evaluated as more likable than an agent who displayed cooperative nonverbal behavior.

Moreover, we tested whether users' perception of the agent prior to the dilemma influenced the user's cooperation behavior during the dilemma game. A multiple linear regression with the first evaluation of Likability, Cooperativity, and Competence as predictors and users' cooperation behavior during the game as dependent variable showed a valid regression model for Likability, $b=.29, t(78)=2.56, p=.013$. Initial Likability ratings also explained a significant proportion of variance in cooperation behavior, $R^{2}=.07, F(1,79)=6.53, p$ $=.013$.

3.3. Communication Abilities. To examine the effect of age group and nonverbal behavior on the perceived communication abilities of the agent, we calculated two-factorial ANOVAs. However, the analyses for the four subscales did not result in any significant differences.

3.4. Future Usage. Results of an ANOVA showed no significant effect of age group or nonverbal behavior on the intended future usage of the agent.

3.5. Perception of the Game. The effect of nonverbal behavior and age group on the perception of the game based on single items was examined using two-factorial ANOVAs.

Significant main effects of age group were obtainable for the quality of the agent's arguments $(F(1,75)=10.388, p=.002$, 
TABLE 2: Means for participants overall decision to cooperate.

\begin{tabular}{|c|c|c|c|c|c|c|}
\hline \multirow[b]{3}{*}{ Nonverbal behavior } & \multicolumn{6}{|c|}{ Age group } \\
\hline & \multicolumn{2}{|c|}{ Students } & \multicolumn{2}{|c|}{ Seniors } & \multicolumn{2}{|c|}{ Overall } \\
\hline & $M$ & $S D$ & $M$ & $S D$ & $M$ & $S D$ \\
\hline Coop & 2.85 & 0.93 & 3.20 & 0.95 & 3.03 & 0.95 \\
\hline Non-coop & 2.55 & 0.83 & 3.35 & 0.59 & 2.95 & 0.81 \\
\hline Overall & 2.70 & 0.88 & 3.28 & 0.78 & 2.99 & 0.88 \\
\hline
\end{tabular}

TABLE 3: Means of age group's game evaluation.

\begin{tabular}{|c|c|c|c|c|c|c|}
\hline \multirow{3}{*}{ Nonverbal behavior } & \multicolumn{6}{|c|}{ Age Group } \\
\hline & \multicolumn{2}{|c|}{ Students } & \multicolumn{2}{|c|}{ Seniors } & \multicolumn{2}{|c|}{ Overall } \\
\hline & $M$ & $S D$ & $M$ & $S D$ & $M$ & $S D$ \\
\hline $\begin{array}{l}\text { Billie provided good arguments for cooperation } \\
\text { during the game }\end{array}$ & 3.23 & 1.25 & 4.05 & 0.96 & 3.64 & 1.18 \\
\hline $\begin{array}{l}\text { Billie gave me the feeling that he knows better and } \\
\text { I am better off not to trust him }\end{array}$ & 2.68 & 1.39 & 2.00 & 1.20 & 2.34 & 1.33 \\
\hline I perceived Billie as my opponent & 2.65 & 1.33 & 1.90 & 1.24 & 2.28 & 1.33 \\
\hline $\begin{array}{l}\text { During the game I was mostly concerned with } \\
\text { how I could maximize my own gains }\end{array}$ & 2.53 & 1.40 & 1.75 & 1.03 & 2.14 & 1.28 \\
\hline $\begin{array}{l}\text { I often thought about how Billie would decide in } \\
\text { this round }\end{array}$ & 4.05 & 0.96 & 2.55 & 1.52 & 3.30 & 1.47 \\
\hline
\end{tabular}

$\left.\eta^{2}=.122\right)$, the perceived trust towards the agent $(F(1,75)=$ 4.731, $\left.p=.033, \eta^{2}=.059\right)$, feelings of competitiveness $(F(1,75)$ $\left.=6.140, p=.015, \eta^{2}=.076\right)$, maximal gain $(F(1,75)=7.50 p$ $\left.=.008, \eta^{2}=.091\right)$, and the agent's decisions $(F(1,75)=28.826, p$ $\left.<.001, \eta^{2}=.278\right)$. Overall, the results showed the pattern that seniors felt less competitive, while students tried to maximize their gain and took into account the agent's potential future decision-making behavior more strongly during their own decision-making (c.f. Table 3).

Additionally, main effects of the agent's nonverbal behavior on the perceived difficulty of the game $(F(1,75)=5.22, p$ $=.025, \eta^{2}=.063$ ) and feeling of being confederates were found $\left(F(1,75)=5.010, p=.028, \eta^{2}=.063\right)$. On the one hand, users perceived the game as being easier when they interacted with an agent that displayed cooperative nonverbal behavior (CO: $M=1.20, S D=0.46$; NC: $M=1.75, S D=1.32$ ); on the other hand, participants perceived the agent more as a confederate when he showed noncooperative nonverbal behavior (CO: $M$ $=2.63, S D=1.28$; NC: $M=3.23, S D=1.10$ ).

3.6. Physical and Social Presence. Further calculations demonstrated that age group and the agent's nonverbal behavior did not affect the perceived copresence of the agent.

3.7. Moderating Variables. We calculated multiple ANCOVAs with personality traits and nonverbal immediacy as moderating variables, but the presented pattern of results did not change.

\section{Discussion, Limitations, and Future Work}

In this study, we experimentally tested the influence of a virtual agent's (non)cooperative nonverbal behavior on users' evaluation of the agent and their willingness to cooperate during a social dilemma game. Eliciting the perception of cooperativeness with nonverbal cues might be used as a subtle way in human-agent interaction to enhance users' willingness to cooperate without forcing them.

Surprisingly, our results did not demonstrate the expected influence of the agent's nonverbal behavior on participants' decision-making. In contrast to prior research and hypothesis $\mathrm{H} 1$, the user's willingness to cooperate during the Prisoner's Dilemma was not affected by the agent's nonverbal behavior. The scenario utilized for this study might have suppressed effects of nonverbal behavior, since the Prisoner's Dilemma is restricted in terms of the length of interaction and quality of interpersonal communication. Hence, participants' focus might have been more on the task than on the agent. In this regard, the manipulation of (non)cooperative nonverbal behavior might have been too subtle for the given scenario.

A manipulation check on whether participants consciously perceived the nonverbal behavior of the agent indeed indicated that participants were not able to report whether the agent showed cooperative behaviors or not. Therefore, participants stated how much the agent has moved, showed gestures, smiled, and looked towards them, and no significant differences between the conditions have been found. Since the mean values of all items were rather low (moved: $M=$ $1.98, S D=.60$; showed gestures: $M=1.85, S D=.66$; smiled: $M=1.56, S D=.65$; looked at me: $M=2.69, S D=.89$ ), results indicate that the nonverbal behavior was not recognized by the participants in the way it was intended. This, however, is not automatically detrimental in our setting as nonverbal behavior might still be effective even if it is not consciously perceived. However, since the agent's nonverbal behavior had also no effect on the perceived cooperativeness of the agent, 
the presented behavior might have been too subtle or participants focused too much on the presented scenario. Therefore, hypothesis $\mathrm{H} 1$ was not supported. To investigate the role of the chosen scenario and its attracted attention in the recognition and perception of the nonverbal behavior, future studies may use eye-tracking methods to check participants eye-movements and focus during the interaction. That would be an objective way, to test whether participants looked at the agent and were able to obtain its nonverbal behavior. Further on, a more social scenario that ensures longer interactions between the agent and the user (as it is true for potential assistive applications, e.g., reviewing a schedule) and offers the option to test cooperation behavior should be used to examine the effect of nonverbal behavior in more detail.

Another possible explanation for our findings might be the context of the nonverbal behavior, because prior research has shown that emotional expressions are affected by the context in which they are presented. Melo and colleagues [18] showed that the morality of the nonverbal behavior is important. For instance, an agent who smiled after a user's loss was perceived as less cooperative than an agent showing empathy and displaying a sad facial expression when the user has lost. Although the agent followed the same scheme for cooperation during the game (yes, no, yes, yes), the cooperative behavior of the respective participant was not foreseeable. Hence, situations might have emerged where the agent displayed smiling behavior after a user's loss. The present study did not focus on the context of the nonverbal behavior, but on its mere exposure.

Besides the effect of the agent's nonverbal behavior, we investigated how the actual cooperation behavior of the agent influenced the user's decisions. We hypothesized that a noncooperative decision of the agent will be followed by a drop in cooperation on the side of the users (H2). Results indicate that the agent's actual decision-making behavior in the Prisoners' Dilemma influenced users' decision-making. While we observed a high initial willingness to cooperate, participants significantly cooperated less after the agent's noncooperative behavior in the second round of the game supporting our hypothesis. These findings are in line with prior research [9], demonstrating that persons are more cooperative when they expect their counterparts to cooperate as well. Thus, after the agent destroyed the participants' trust by being not cooperative, participants did not expect the agent to be cooperative anymore and therefore their own willingness to cooperate also decreases.

Additionally, likability ratings prior to the game predicted participants' willingness to cooperate. Participants who perceived the agent as more likable before they played the game cooperated more with the agent. The likability perception after the game was affected by the agent's nonverbal behavior, but not in the way as it was intended, since noncooperative behavior led to higher likability ratings. Although the nonverbal behavior was chosen based on prior research [4], where those behaviors evoked a higher cooperativeness and likability perception, this pattern could not be replicated in the present study. During the used scenario showing only little nonverbal behavior seemed to be perceived as more likable than being nonverbal expressive. Maybe the cooperative behaviors were seen as not appropriate while gambling for money. However, this finding is contradicting to our assumptions and the empirical background.

Since applications of virtual agents for people in need of support (like the target group of seniors) are supposed to be beneficial and since cooperation in these contexts is regularly needed in order to provide benefits, we examined age-related differences in the perception of cooperativeness and the user's intention to cooperate. Our results demonstrate that seniors cooperated more often with the agent than students did. In addition, results regarding the evaluation of the game revealed that seniors showed unconditional trust and cooperation to the agent, since they stated to have less competitive thoughts. In contrast, students were more competitive and tried to maximize their win by considering the agent's behavior more carefully. No differences with regard to the perception of the agent's cooperativeness have been found. Seniors and students evaluated the agent similarly. Moreover, no interaction effects of age group and nonverbal behavior have been found. Therefore, the effect of the nonverbal behavior did not differ between the age groups and both groups perceived the agent in the same way.

\section{Conclusion}

It can be concluded that while actual cooperative behavior as well as the evaluation of the game was influenced by age, the perception and evaluation of the agent's nonverbal behavior were not. In summary, findings of the present study suggest that the actual decision-making behavior of a virtual agent is more important than the agent's nonverbal behavior-at least in this specific setting of the Prisoner's Dilemma. For a money-based game, the nonverbal behavior seemed to be too subtle to unfold full effect. An additional important finding with regard to future applications is that seniors cooperated more often with the agent and showed unconditional trust. This might be helpful with regard to future applications in which agents and humans cooperate to the human's benefit.

\section{Data Availability}

All relevant data are within the paper and the data set can be requested from the corresponding author.

\section{Conflicts of Interest}

The authors declare that there are no conflicts of interest regarding the publication of this paper.

\section{Acknowledgments}

This work was part of the project KOMPASS (socially cooperative, virtual assistants as companions for people in need of cognitive support) [Grant no. 16SV7272] that is funded by the German Federal Ministry of Education and Research. The authors would like to thank Ramin Yaghoubzadeh, Katharina Suhre, Katharina Brockmann, and Sueyda Yüzer for their help with the scenario implementation and data collection. 
They further acknowledge support by the Open Access Publication Fund of the University of Duisburg-Essen.

\section{References}

[1] R. Yaghoubzadeh, M. Kramer, K. Pitsch, and S. Kopp, "Virtual agents as daily assistants for elderly or cognitively impaired people," in Proceedings of the International Conference on Intelligent Virtual Agents, pp. 79-91, 2013.

[2] L. I. Reed, K. N. Zeglen, and K. L. Schmidt, "Facial expressions as honest signals of cooperative intent in a one-shot anonymous Prisoner's Dilemma game," Evolution and Human Behavior, vol. 33, no. 3, pp. 200-209, 2012.

[3] R. Thomas Boone and R. Buck, "Emotional expressivity and trustworthiness: The role of nonverbal behavior in the evolution of cooperation," Journal of Nonverbal Behavior, vol. 27, no. 3, pp. 163-182, 2003.

[4] C. Straßmann, A. R. Von Der Pütten, R. Yaghoubzadeh, R. Kaminski, and N. Krämer, "The effect of an intelligent virtual agent's nonverbal behavior with regard to dominance and cooperativity," in Proceedings of the 16th International Conference on Intelligent Virtual Agents (IVA '16), pp. 15-28, Los Angeles, Calif, USA, September 2016.

[5] E. Krumhuber, A. S. R. Manstead, D. Cosker, D. Marshall, P. L. Rosin, and A. Kappas, "Facial dynamics as indicators of trustworthiness and cooperative behavior," Emotion, vol. 7, no. 4, pp. 730-735, 2007.

[6] W. M. Brown and C. Moore, "Smile asymmetries and reputation as reliable indicators of likelihood to cooperate: An evolutionary analysis," Advances in Psychology Research, vol. 11, no. 3, pp. 59-78, 2002.

[7] J. Jahng, J. D. Kralik, D.-U. Hwang, and J. Jeong, "Neural dynamics of two players when using nonverbal cues to gauge intentions to cooperate during the Prisoner's Dilemma Game," NeuroImage, vol. 157, pp. 263-274, 2017.

[8] J. Schug, D. Matsumoto, Y. Horita, T. Yamagishi, and K. Bonnet, "Emotional expressivity as a signal of cooperation," Evolution and Human Behavior, vol. 31, no. 2, pp. 87-94, 2010.

[9] J. L. Pletzer, D. Balliet, J. Joireman, D. M. Kuhlman, S. C. Voelpel, and P. A. M. Van Lange, "Social value orientation, expectations, and cooperation in social dilemmas: a meta-analysis," European Journal of Personality, vol. 32, no. 1, pp. 62-83, 2018.

[10] N. C. Krämer, L. Hoffmann, and S. Kopp, "Know your users! Empirical results for tailoring an agent's nonverbal behavior to different user groups," in Proceedings of the International Conference on Intelligent Virtual Agents, vol. 6356, pp. 468-474, 2010.

[11] J. M. Beer, C. Smarr, A. D. Fisk, and W. A. Rogers, "Younger and older users' recognition of virtual agent facial expressions," International Journal of Human-Computer Studies, vol. 75, pp. 1-20, 2015.

[12] A. M. Rosenthal-Von Der Pütten, N. Bock, and K. Brockmann, "Not your cup of tea?: How interacting with a robot can increase perceived self-efficacy in HRI and evaluation," in Proceedings of the 12th Annual ACM/IEEE International Conference on Human-Robot Interaction (HRI '17), pp. 483-492, March 2017.

[13] K. L. Nowak and F. Biocca, "The Effect of the Agency and Anthropomorphism on users' Sense of Telepresence, Copresence, and Social Presence in Virtual Environments," Presence: Teleoperators and Virtual Environments, vol. 12, no. 5, pp. 481494, 2003.
[14] J. N. Bailenson, A. C. Beall, J. Blascovich, M. Raimundo, and M. Weisbuch, 'Intelligent agents who wear your face: Users' reactions to the virtual self," in Proceedings of the International Conference on Intelligent Virtual Agents, vol. 2190, pp. 86-99, 2001.

[15] B. Rammstedt and O. P. John, "Measuring personality in one minute or less: a 10-item short version of the big five inventory in English and German," Journal of Research in Personality, vol. 41, no. 1, pp. 203-212, 2007.

[16] Black Dog Institute, “Temperament \& personality (T\&P) questionnaire information," BlackDog Insitution, 2012, http://www .blackdoginstitute.org.au/.

[17] V. P. Richmond, J. C. McCroskey, and A. D. Johnson, "Development of the nonverbal immediacy scale (NIS): Measures of self-and other-perceived nonverbal immediacy," Communication Quarterly, vol. 51, no. 4, pp. 504-517, 2003.

[18] C. M. De Melo, L. Zheng, and J. Gratch, "Expression of moral emotions in cooperating agents," in Proceedings of the International Conference on Intelligent Virtual Agents, pp. 301$307,2009$. 


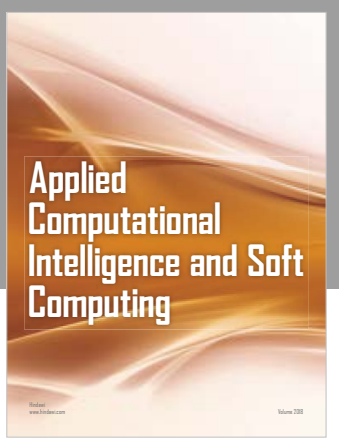

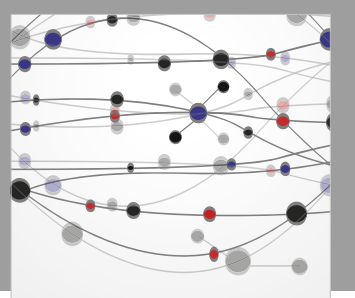

The Scientific World Journal
Submit your manuscripts at

Computing
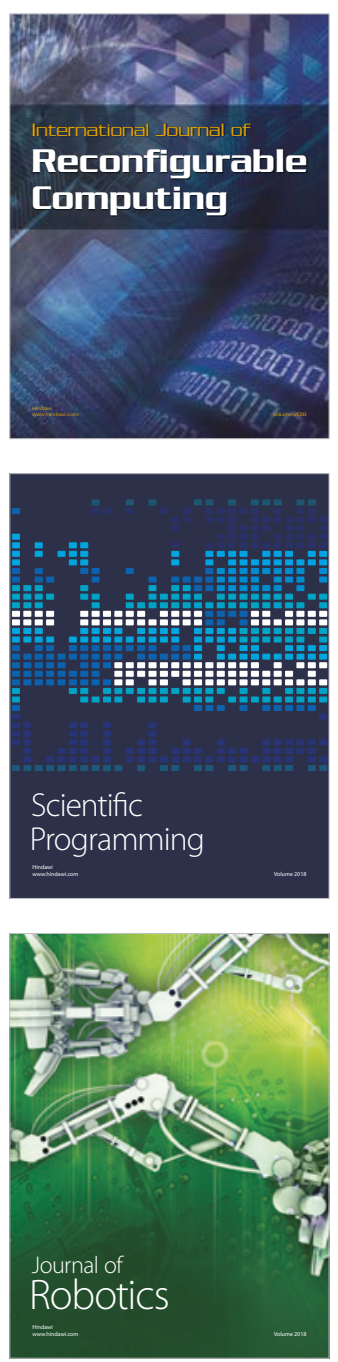

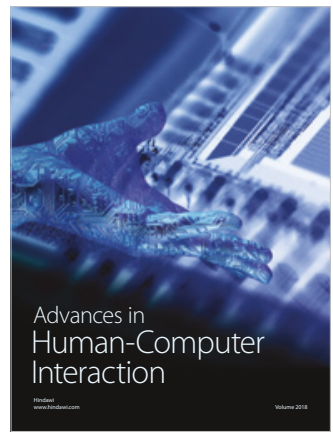

Human-Compute

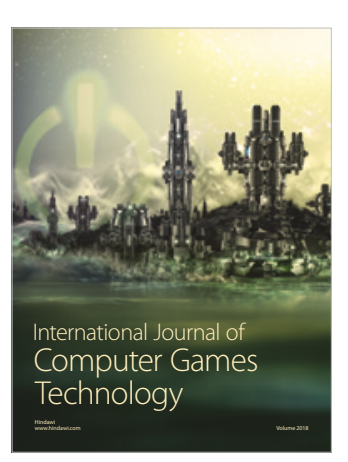

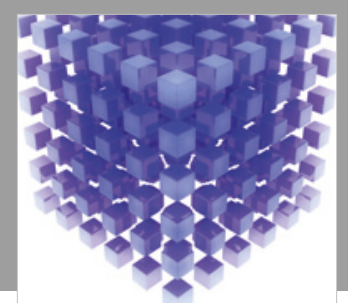

Mathematical Problems in Engineering

\section{Engincering}
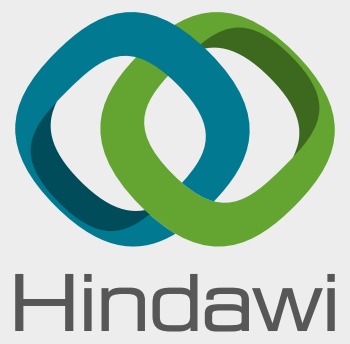

www.hindawi.com
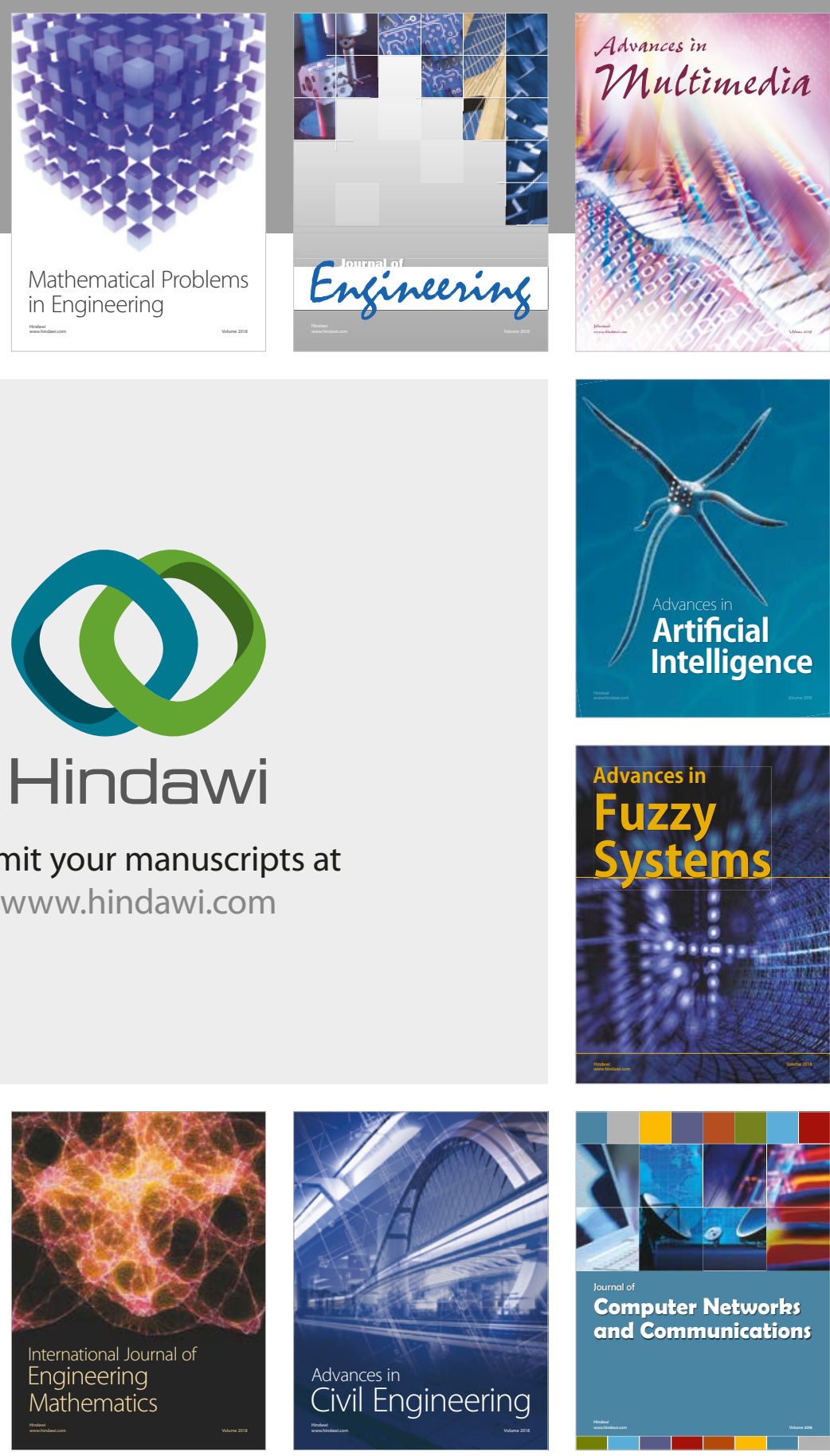

Computer Networks and Communications

Multimedia
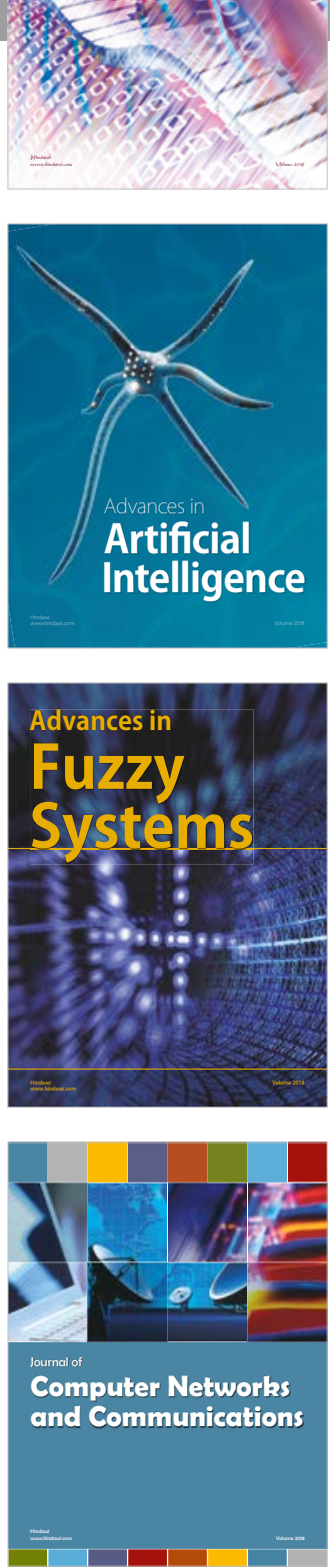

Advances in

Modelling \&

Simulation

in Engineering

interaction

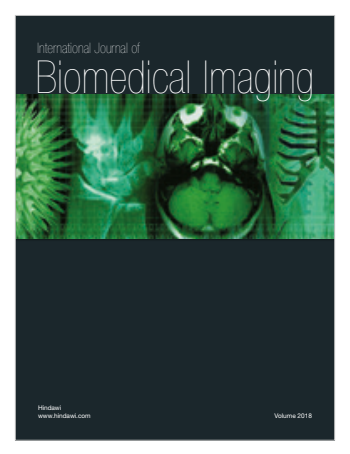

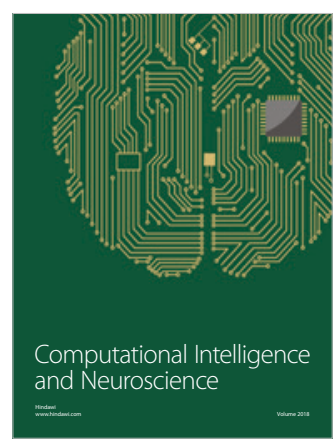

